J Ästhet Chir 2019 · 12:22-29 https://doi.org/10.1007/s12631-018-0167-y Online publiziert:6. Dezember 2018

(c) Der/die Autor(en) 2018

CrossMark

Karsten Knobloch

SportPraxis Prof. Knobloch, Hannover, Deutschland

\title{
Cellulitebehandlung mithilfe von Stammzellen und Stoßwellen
}

\section{Was kann man mit physikalischen Methoden erreichen?}

lenkt, um den Stein zu zertrümmern. Die Trümmerteile fanden dann den Weg via naturalis ans Körperäußere. In der Folge wurden weitere Nierensteinzertrümmerungen (Lithotripsien) auf diese Weise erfolgreich durchgeführt. Damit fand die ESWT zunächst in den urologischen Abteilungen in Deutschland ihre Bestimmung.

\section{Mechanismus der extra- korporalen Stoßwellentherapie}

Die initialen frühen, v. a. auch urologischen Erfahrungen mit der ESWT entwickelten eine mechanistische Hypothese hinsichtlich der Wirkweise: Je höher die ESWT-Energie, desto wahrscheinlicher konnte diese z. B. einen Nierenstein zertrümmern. Je mehr Energie, so ein Gedanke, desto größer der klinische Effekt in Bezug auf die Nierensteinzertrümmerung.

Diese urologisch verwendeten sehr hohen Energieflussdichten machten All- gemeinnarkosen bei der Nierensteinzertrümmerung notwendig. Im weiteren Verlauf und mit wachsender Stoßwellenerfahrung wurden dann zunächst die Unfallchirurgen auf die ESWT aufmerksam, weil u.a. Pseudarthrosen durch die ESWT zur klinischen und radiologischen Abheilung gebracht werden konnten. Mittlerweile darf bei Pseudarthrosen in der Hand des sachkundigen ESWT-Arztes mit Erfolgsraten um $80 \%$ gerechnet werden.

Beachtet man beispielsweise die berichteten Energieflussdichten (EFD) der klinischen ESWT-Studien über die letzten 10 Jahre im internationalen Vergleich, so fällt auf, dass die verwendeten Energieflussdichten sukzessive zurückgingen, während die klinisch bemerkten Effekte mitunter sogar stiegen - mit anderen Worten bei reduzierter Energie ein verbesserter klinischer Effekt (• Abb. 1). Diese Beobachtung wie auch jüngere experimentelle Ergebnisse legen eine regenerative Wirkung mit deutlich niednet sich durch eine besondere Kurvenform mit steiler Anstiegsgeschwindigkeit aus. Es ist dann dem Einfallsreichtum und der Weitsicht von Luft- und Raumfahrtingenieuren zu verdanken, dass die Stoßwellentherapie den Weg in die Medizin fand.

Am 07.02.1980 fand die erste klinische Stoßwellentherapiesitzung im Klinikum Großhadern statt. Ein Patient mit einem schmerzhaften Nierenstein wurde in einem Wasserbad in Narkose gebadet. Die Stoßwelle wurde hochenergetisch außerhalb des Körpers (daher extrakorporal) generiert und gezielt auf das Nierensteinlager unter Röntgensicht ge-

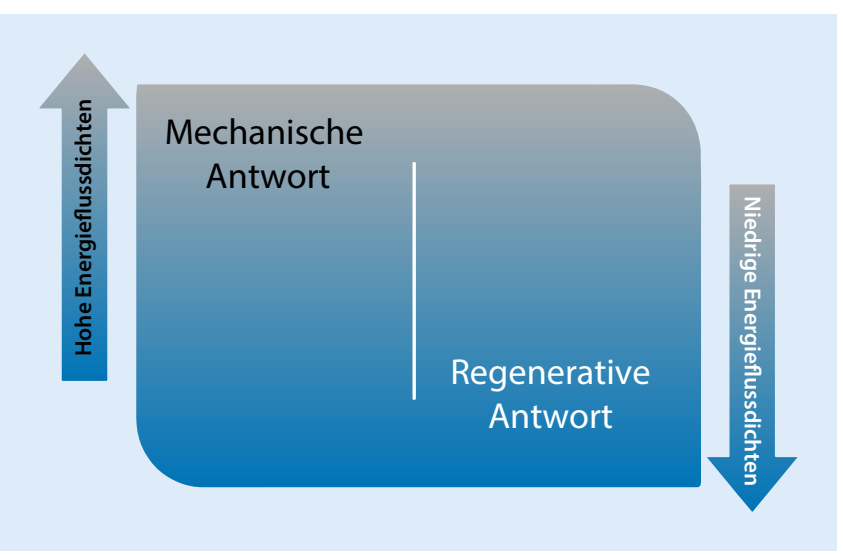

Abb. 1 Während hohe Energieflussdichten wie bei der Lithotripsie zur Nierensteinzertrümmerung im Wesentlichen eine mechanische Antwort auslösen, führen niedrige Energieflussdichten zu einer regenerativen Gewebeantwort 


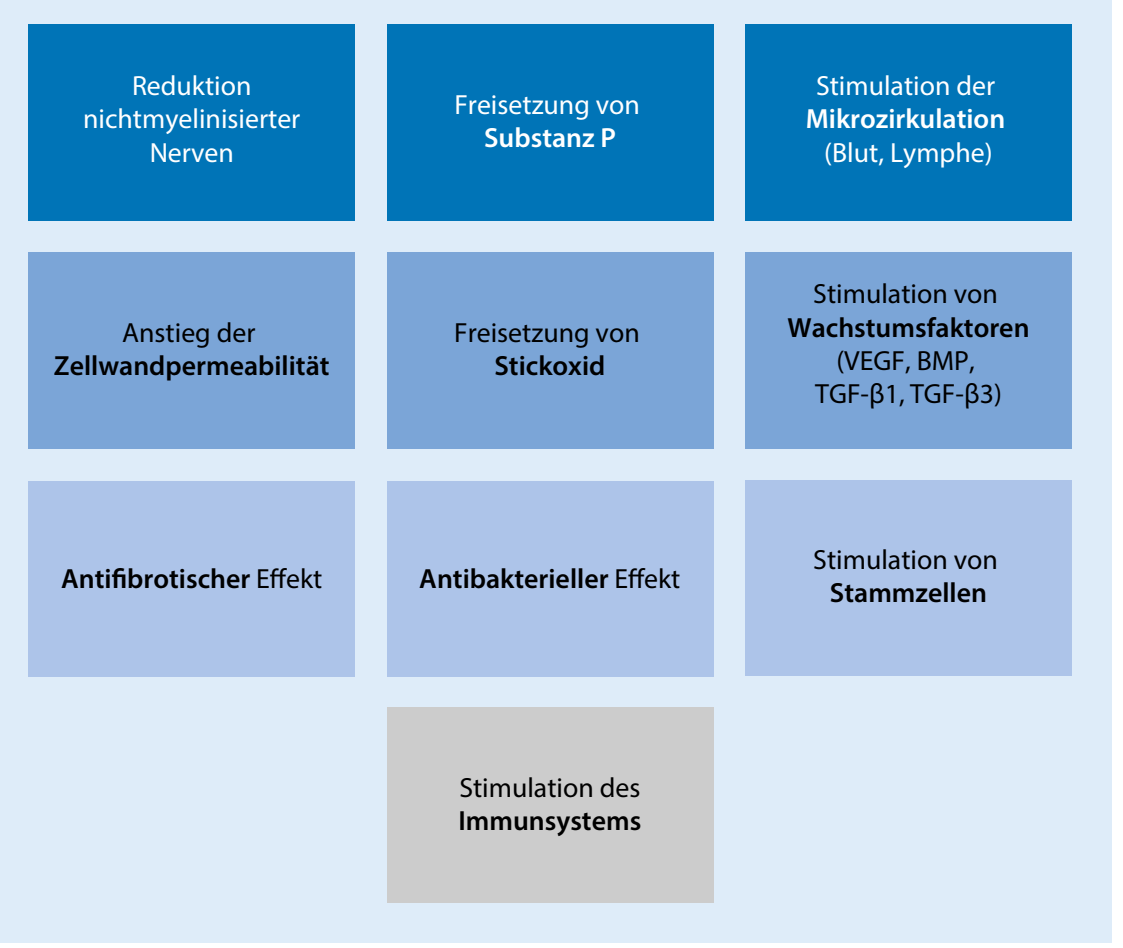

Abb. 2 A Bislang beschriebene biologische Effekte der extrakorporalen Stoßwellentherapie (ESWT) im Gewebe

rigeren Energieflussdichten im Vergleich zur mechanistischen Steinzertrümmerung nahe.

Johannes Holfeld [1] aus der herzchirurgischen Universitätsklinik Innsbruck konnte mit seiner Arbeitsgruppe u.a. nachweisen, dass die fokussierte Stoßwellentherapie eine Stammzellaktivierung auslöst. Darüber hinaus ist bis heute eine Reihe an weiteren biologischen Effekten durch die ESWT beschrieben, die auch bei der Cellulite eine Rolle spielen können, wie beispielsweise die Verbesserung der Mikrozirkulation oder auch die Stimulation von Lymph-VEGF (,lymphatic vascular endothelial growth factor") wie auch ein antifibrotischer Effekt (• Abb. 2).

\section{I) Die fokussierte Stoß- wellentherapie löst eine Stammzellaktivierung aus}

Wie angesprochen kann die ESWT dosis- und geräteabhängig zelluläre Effekte erzielen. In einer Dosis-Wirkungs-Studie aus China [2] wurden unterschiedliche Energieflussdichten von

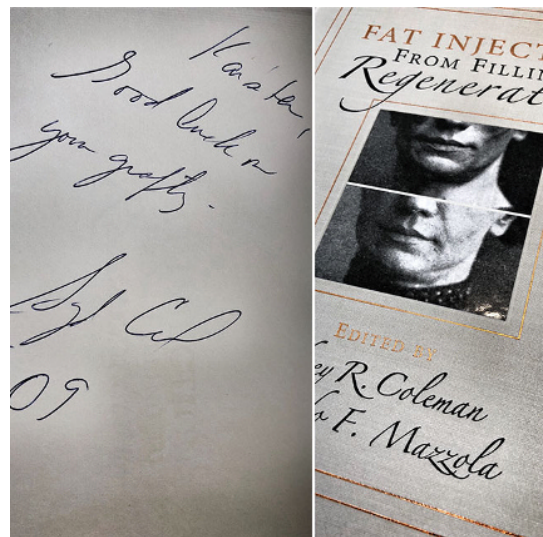

Abb. 3 A Persönliches Exemplar des Standardwerks der Fetttransplantationsprotagonisten Sydney Coleman und Riccardo Mazzola: Fat injection - from filling to regeneration, veröffentlicht 2009

durch die Verfeinerung und Systematisierung des autologen Fetttransfers mit Protagonisten wie Sydney Coleman [4] konnten die Resorptionsraten deutlich reduziert und das klinische Potenzial konnte deutlich verbessert werden (• Abb. 3). In den letzten 15 Jahren kam es gewissermaßen zur einer Renaissance der autologen Fetttransplantation.

Im deutschsprachigen Raum fand im September 2009 in Hannover eine erste Konsensuskonferenz zu den Chancen und Risiken der autologen Fetttransplantation unter der Federführung der DGPRÄC (Deutsche Gesellschaft der Plastischen, Rekonstruktiven und Ästhetischen Chirurgen) statt, deren Ergebnisse auch entsprechend publiziert wurden [5]. Regulatorisch, so die Konsensusentscheidung, ist das in Deutschland gültige Gewebegesetz einzuhalten. Für die Vergleichbarkeit der Ergebnisse der autologen Fetttransplantation, so die Konsensusentscheidung, seien die Details zur Entnahme-, der Verarbeitungswie auch der Reinjektionstechnik essenziell. Schon 2009 schloss die Konferenz, dass neue Techniken wie die stamm- und stromazellangereicherte Fetttransplantation möglicherweise in der Zukunft weitere Indikationen eröffne.

Der Stellenwert der autologen Fetttransplantation in der Senologie wurde in diesem Journal von Frau Dr. Rezek lesenswert vorgestellt [6]. 
Der autologe Fetttransfer als operative Prozedur beinhaltet (- Abb. 4):

- die Fettgewinnung per Liposuktion,

- die Aufbereitung des gewonnenen Lipoaspirates außerhalb des Körpers und

- die schlussendliche autologe Fetttransplantation.

Die Fettgewinnung mit Liposuktion kann nachhaltig Einfluss auf die Qualität der Fettstammzellen nehmen. So ist der Absaugdruck bei der Liposuktion möglichst niedrig zu halten. Eine chinesische Arbeitsgruppe [7] zeigte beispielsweise, dass $-30 \mathrm{kPa}$ im Vergleich zu -55 kPa Absaugdruck eine deutlich bessere Zellüberlebenswahrscheinlichkeit mit höheren Anteilen von "fibroblast growth factor" und VEGF sowie eine verbesserte adipogene Differenzierung erzielt.

\section{Extrakorporale Stoßwellentherapie bei autologer Fetttransplantation}

Im März 2013 veröffentlichte eine Arbeitsgruppe aus Gießen einen ersten Bericht zum positiven Einfluss der ESWT auf „adipose-derived stem cells“ (ASCs) von Pferden [8]. Die fokussierte ESWT verbesserte die Proliferation und Expression von Connexin 43 und aktivierte (phosphorylierte) Erk1/2.

Im Jahr 2014 publizierte eine Arbeitsgruppe vom Ludwig Boltzmann Institut in Wien eine Untersuchung zum Einfluss der ESWT auf ASCs [9]. Dabei wiesen sie bei humanen wie auch Ratten-ASCs eine nachhaltige positive Beeinflussbarkeit mit Erhöhung von mesenchymalen Markern wie CD73, CD90 und CD105 wie auch eine verbesserte Differenzierung in Richtung osteogener und adipogener Zelllinien durch die ESWT nach.

\begin{tabular}{l}
\hline Eine „präventive“ ESWT vor \\
Lipotransfer im Rahmen der \\
autologen Fetttransplantation ist \\
sinnvoll
\end{tabular}

Im September 2017 wurden aus selbiger Arbeitsgruppe Ergebnisse zum Einfluss der niedrigenergetischen ESWT auf die stromale vaskuläre Fraktion (SVF) ver-

J Ästhet Chir 2019 · 12:22-29 https://doi.org/10.1007/s12631-018-0167-y

(c) Der/die Autor(en) 2018

\section{K. Knobloch}

\section{Cellulitebehandlung mithilfe von Stammzellen und Stoßwellen. Was kann man mit physikalischen Methoden erreichen?}

\section{Zusammenfassung}

Die extrakorporale Stoßwellentherapie (ESWT) bedient sich akustischer Wellen, um biologische Wirkungen im Zielgewebe zu erreichen. Seit ihrer ersten klinischen Anwendung im Jahr 1980 ist eine Reihe an biologischen Antworten der ESWT mechanistisch aufgeklärt worden, so u. a. eine Stammzellaktivierung, antifibrotische Effekte, Effekte auf den verbesserten Lymphabfluss und weitere mehr. Zur Cellulite liegen mithin 12 klinische kontrollierte Studien vor, die positive Effekte der ESWT mit radialen wie auch fokussierten ESWT-Technologien bestätigen. Auch die Stimulation von autologem Fettgewebe mit ", adipose-derived stem cells" durch die ESWT ist mit einigen experimentellen Studien belegt. Insofern erscheint die ESWT als nebenwirkungsfreie Therapieform in der Hand des sachkundigen ESWT-Anwenders eine hochattraktive Therapieform zu sein, die auch in der Kombination mit anderen Therapieverfahren sinnvoll ist.

Schlüsselwörter

Extrakorporale Stoßwellentherapie · Autologe Fetttransplantation - Lymphabfluss .

Stammzellaktivierung $\cdot$ Nebenwirkungsfrei

\section{Cellulitis treatment with stem cells and shock waves. What can be achieved with physical methods?}

\section{Abstract}

Extracorporeal shock wave therapy (ESWT) uses acoustic waves to elicit a biological cellular response. Since its first clinical application in 1980 several biological responses of a given tissue to ESWT have been elucidated, such as a stem cell activation, antifibrotic effects and enhancement of lymph drainage. As far as cellulitis is concerned 12 clinically controlled studies are available that confirmed the positive effects of both radial and focused ESWT on cellulitis. Stimulation of autologous fatty tissue with adipose-derived stem cells (ASC) by ESWT has recently been shown experimentally. Thus, ESWT appears to be a highly attractive noninvasive therapeutic option without adverse effects when performed by an experienced and certified ESWT therapist.

\section{Keywords}

Extracorporeal shock wave therapy . Autologous fat transplantation - Lymph drainage $\cdot$ Stem cell activation $\cdot$ No side effects öffentlicht [10]. Die stromale vaskuläre Fraktion ist ein heterogener Mix aus Fibroblasten, Lymphozyten, Monozyten/Makrophagen, endothelialen Zellen, endothelialen Vorläuferzellen, Perizyten und ,adipose-derived strome/stem cells“ (ASC). Nach ESWT auf das humane Fettgewebe konnten signifikant höher zelluläre $\operatorname{ATP}$ (Adenosintriphosphat)-Spiegel nachgewiesen werden als nach ESWT auf die stromale vaskuläre Fraktion. Des Weiteren zeigte die ESWT auf humanem Fettgewebe eine höhere Expression von mesenchymalen und vaskulären Biomarkern als die Kontrollgruppe. Die detaillierte Analyse der stromalen vaskulären Fraktion nach ESWT zeigte eine signifikante Erhöhung von „insulin-like growth factor 1“(IGF)-1 und „placental growth factor" (PLGF). In einer weiteren Arbeit zeigte diese Arbeitsgruppe [11], dass die ESWT in situ die Zellüberlebensrate im Lipoaspirat erhöht und die adipogene Differenzierung verstärkt.

Folgt man dieser Beobachtung, so macht eine „präventive“ ESWT vor Lipotransfer im Rahmen der autologen Fetttransplantation Sinn, um entsprechend die Zellüberlebensrate und die Qualität des Lipoaspirates zu verbessern (•Abb. 5). 


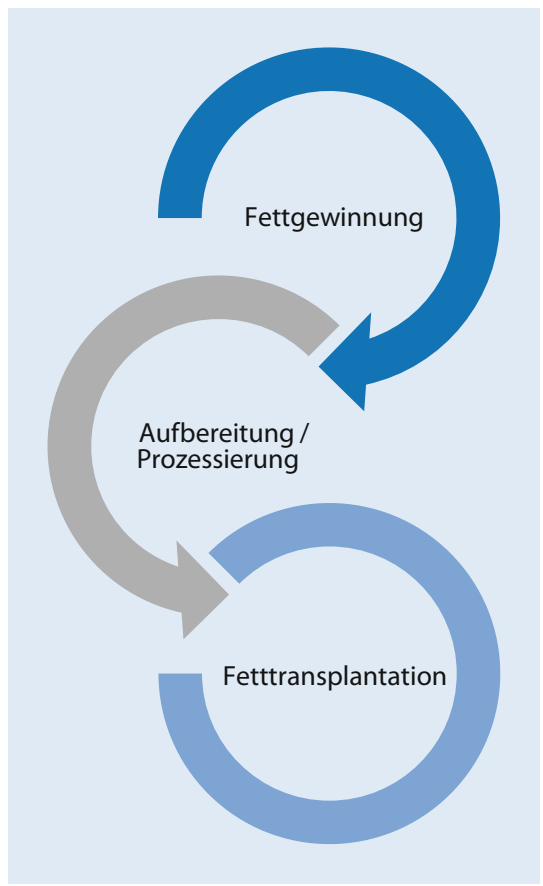

Abb. 4 \& Konventionelle Sequenz bei autologer Fetttransplantation mit Fettgewinnung durch Liposuktion, extrakorporale Aufbereitung/Prozessierung des Lipoaspirates und schließlich autologe Fetttransplantation als finalem Prozessschritt

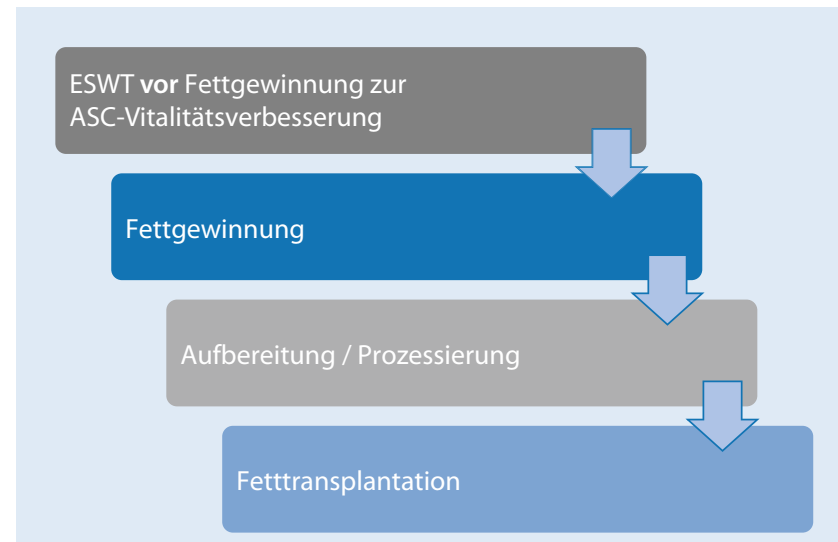

Abb. $5<$ Integration der präkonditionierenden extrakorporalen Stoßwellentherapie (ESWT) vor Fettgewinnung zur Verbesserung der ASC(„adipose-derived stem cell")-Fraktion bei autologer Fetttransplantation

\section{Klinische Bedeutung von "adipose-derived stem cells"}

Interessanterweise beschäftigte sich dieselbe Arbeitsgruppe aus dem Ludwig Boltzmann Institut in Wien auch mit der stromalen vaskulären Fraktion von Lipödempatientinnen [12]. Bei 30 Lipödempatientinnen zeigte die Analyse der stromalen vaskulären Fraktion eine erhöhte Expression der mesenchymalen
Oberflächenproteine CD90 und CD146. Schon 2013 zeigte eine römische Arbeitsgruppe [13], dass bei Diabetes u.a. die ASC-Funktion reduziert ist mit reduzierter Proliferation und limitierter Migration.

\section{Hier steht eine Anzeige.}

\section{Springer}




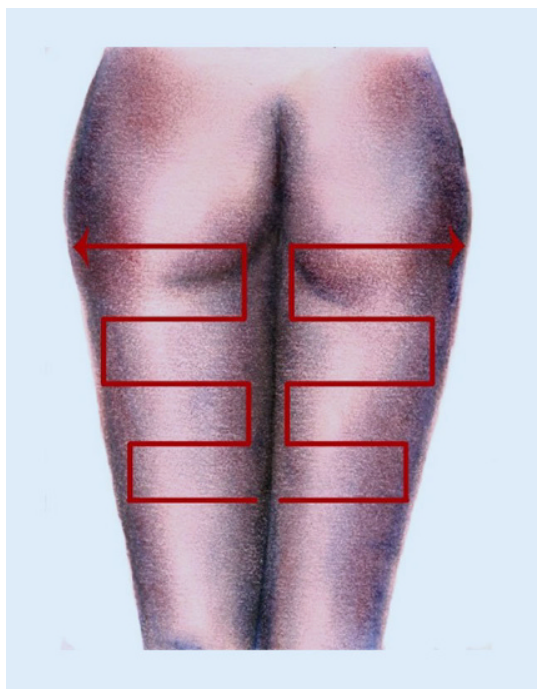

Abb. 6 ॥ Schema der standardisierten fokussierten ESWT(extrakorporale Stoßwellentherapie)-Applikation mit 0,35 $\mathrm{mJ} / \mathrm{mm}^{2} \mathrm{mit}$ 1000 Impulsen pro Seite und Sitzung in der CelluShockStudie von Knobloch et al. (@ K. Knobloch, alle Rechte vorbehalten. Abdruck mit freundl. Genehmigung)

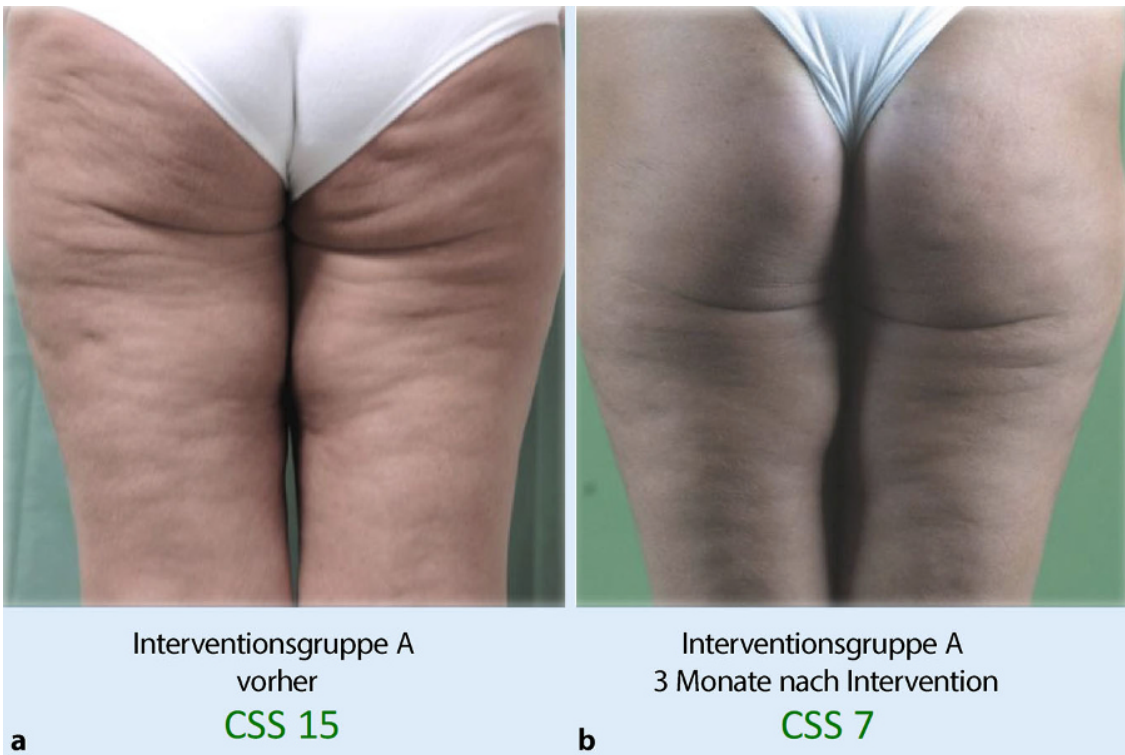

Abb. $7 \Delta$ CelluShock-RCT (randomisiert kontrollierte Studie) zur fokussierten extrakorporalen Stoßwellentherapie (ESWT) a vorher und $\mathbf{b} 3$ Monate nach 6 wöchentlichen fokussierten ESWT-Sitzungen $\left(0,35 \mathrm{~mJ} / \mathrm{mm}^{2}, 2000 \mathrm{Impulse}\right.$, Storz Duolith, Knobloch Ket al.). (@ K. Knobloch, alle Rechte vorbehalten. Abdruck mit freundl. Genehmigung)

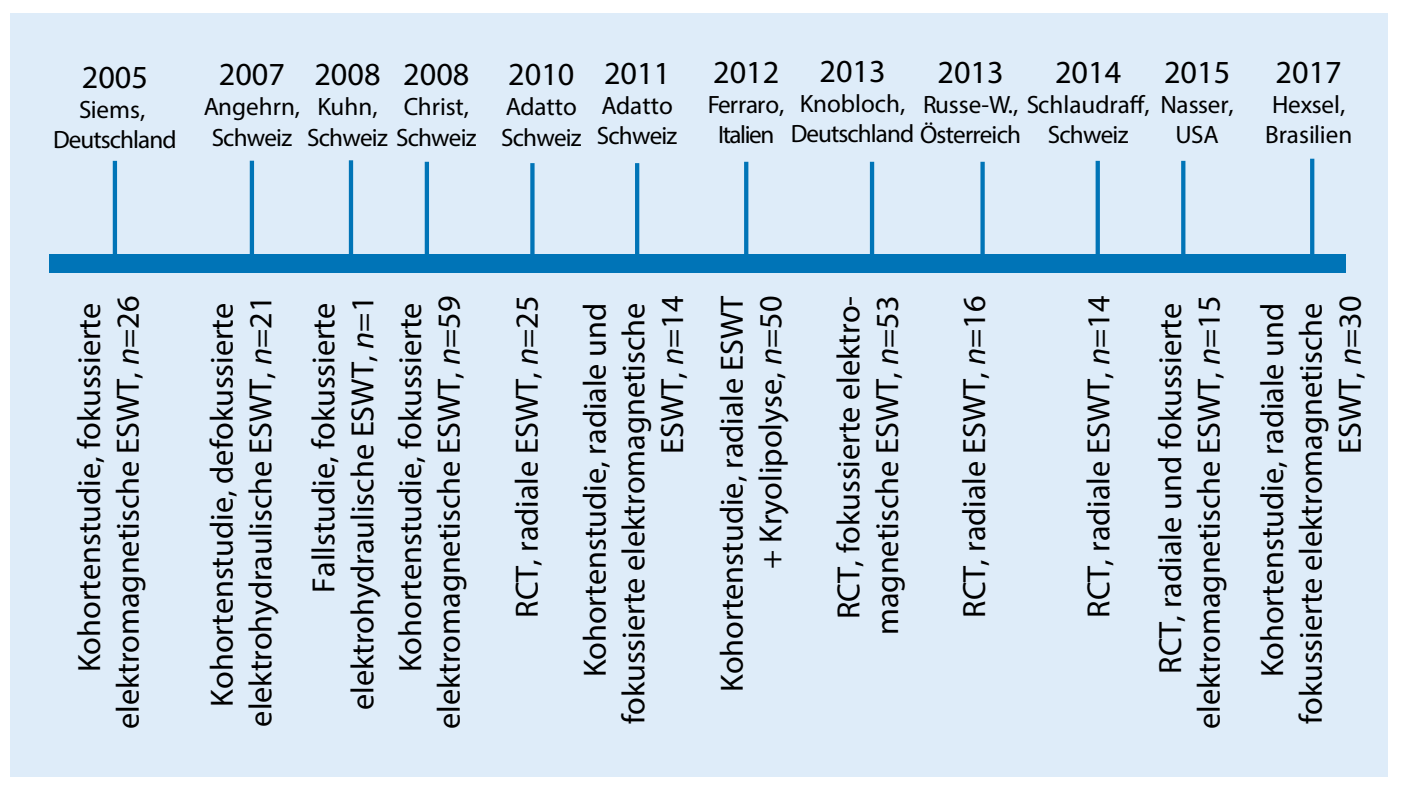

Abb. $8<$ Zeitverlauf der ESWT(extrakorporale Stoßwellentherapie)-Studien zur Cellulite

\section{Extrakorporale Stoßwellen- therapie bei Cellulite - klinische Daten}

Die Cellulite kann sowohl mit der radialen wie auch der fokussierten ESWT positiv verändert werden. Die erste Publikation [14] datiert auf das Jahr 2005, als der seinerzeit Bad Harzburger Kollege Siems insgesamt 26 Patientinnen mit Lipödem und Cellulite mit fokussierter ESWT behandelte. Dabei konnte Siems u. a. nach- weisen, dass die erhöhten Konzentrationen von Serum-Malondialdehyd (MDA) sowie von Produkten der Lipidperoxidation signifikant durch die ESWT reduziert werden konnten. Des Weitern zeigte Siems erstmalig einen engen Zusammenhang der Anzahl der ESWT-Behandlungen und der klinischen Effektivität: Je häufiger behandelt wurde (in der Spanne von 1 bis 6 Sitzungen), desto besser war die gemessene Elastizität der Patientinnenhaut.
In einer eigenen doppelblinden randomisiert kontrollierten Studie (CelluShockStudie) [15] wurden 53 Patientinnen im mittleren Alter von $42 \pm 5$ Jahren (BMI [Body-Mass-Index] 24,2 $\pm 2,3 \mathrm{~kg} / \mathrm{m}^{2}$ ) randomisiert der Interventions- bzw. der Kontrollgruppe zugeteilt. Die Probandinnen der Interventionsgruppe unterzogen sich einer 6-maligen fokussierten Stoßwellentherapie (elektromagnetische fokussierte ESWT, Storz Duolith, Storz Medical, Tägerwilen, Schweiz, Energie- 

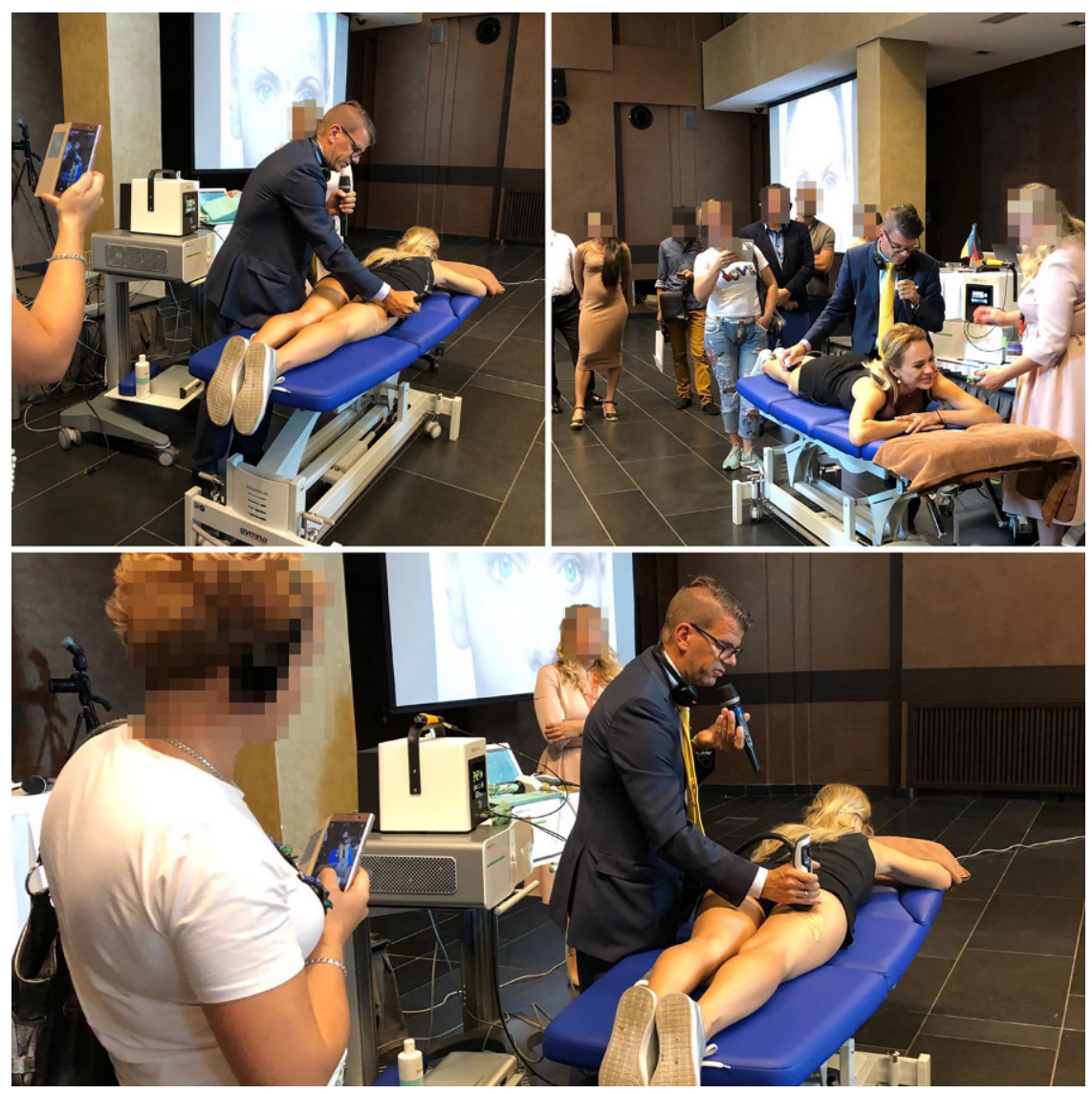

Abb. 9 A Internationale ESWT(extrakorporale Stoßwellentherapie)-Trainingskurse mit radialer und fokussierter ESWT-Technologie zum Transfer der praktischen ESWT-Kompetenz. (@ K. Knobloch, alle Rechte vorbehalten. Abdruck mit freundl. Genehmigung)

flussdichte $0,35 \mathrm{~mJ} / \mathrm{mm}^{2}, 2000$ Impulse pro Sitzung) und einem täglichen selbstständigen glutealen Krafttraining in Form von 2 Übungen. Die Kontrollgruppe führte das identische Krafttraining aus und erhielt 6-malig eine SHAMESWT mit $0,01 \mathrm{~mJ} / \mathrm{mm}^{2}$. Das Follow-up erfolgte 12 Wochen nach den 6 fokussierten Stoßwellensitzungen. Primärer Studienendpunkt war der „cellulite severity score" (CSS), ein validierter Score zur Beurteilung von digitalen Befunden mit einer Spanne von 0 (keine Cellulite) bis 15 Punkten (maximale Cellulite) anhand von standardisiert erfassten digitalen Fotografien ( $\bullet$ Abb. 6).

In der Interventionsgruppe verbesserte sich der CSS von $10,9 \pm 3,8$ vorher auf $8,3 \pm 4$ Punkte nach 12 Wochen $(p=0,001 ; 2,53$ Punkte Verbesserung; 95\%-KI [Konfidenzintervall]: 1,43-3,62 Punkte). Der CSS in der Placebogruppe blieb unverändert bei $10,0 \pm 3,8$ Punkten $(p=0,876 ; 95 \%$-KI:
1,1-0,97 Punkte). Die Veränderung des CSS zwischen der Interventions- und der Kontrollgruppe war hochsignifikant unterschiedlich $(p=0,001 ;-24,3 \%$ Effektgröße; $95 \%$-KI: $-36,5 \%$ bis $-12,1 \%$ ). Einzig in der Interventionsgruppe verbesserten sich alle 5 Unterpunkte der CSS. Es wurden keine Nebenwirkungen beobachtet (• Abb. 7).

》) Die Cellulite kann sowohl mit der radialen wie auch der fokussierten ESWT positiv verändert werden

In einer systematischen Metaanalyse [16] aller publizierter ESWT-Studien zur Cellulite im Jahr 2015 schlossen wir insgesamt 11 Studien mit 297 Patientinnen ein (• Abb. 8).

Von den 11 Studien waren 5 in randomisiert kontrolliertem Studiendesign
(LoE 1b). Sowohl radiale wie auch fokussierte ESWT-Technologien fanden in den 11 Studien Anwendung mit unterschiedlichen Zielparametern und daher einer gewissen Heterogenität. Dennoch lässt sich anhand der vorliegenden publizierten klinischen Studien ableiten, dass die ESWT-Studien

- sowohl mit radialen wie auch mit fokussierten Stoßwellentechnologien erfolgreich waren,

- typischerweise 6 bis 8 ESWT-Sitzungen insgesamt und 1 bis 2 Sitzungen pro Woche umfassten,

- der Nachuntersuchungszeitraum dabei bis zu 6 Monaten betrug.

Mittlerweile ist durch Doris Hexsel aus Porto Allegre in Brasilien im Jahr 2017 eine weitere Kohortenstudie mit 30 eingeschlossenen Frauen veröffentlicht [17]. Es wurde insgesamt 12-malig, 2-mal/ Woche kombiniert radial und fokussiert mit ESWT behandelt. Der mittlere CSS verbesserte sich signifikant von 11,1 auf 9,5 Punkte. Der Hüftumfang reduzierte sich von $103 \mathrm{~cm}$ auf $100 \mathrm{~cm}$ und die Unterhautdicke von $28,3 \pm 7 \mathrm{~mm}$ auf $26,6 \mathrm{~mm}$ ebenfalls signifikant. Etwa $90 \%$ der Frauen waren mit der kombinierten radialen und fokussierten ESWT zufrieden und würden sich dieser Therapie erneut für die Behandlung der Cellulite unterziehen.

\section{Fachkunde Stoßwellentherapie (ESWT)}

Wie es für die chirurgische praktische Ausbildung am Skalpell eine Lernkurve gibt, so ist diese Lernkurve auch bei der Anwendung der Stoßwellentherapie vorhanden. Schon 2014 initiierte die deutschsprachige internationale Gesellschaft für extrakorporale Stoßwellentherapie (DIGEST e.V.) eine 60-stündige Fachkunde "Stoßwellentherapie“ für Fachärzte in Deutschland und kurze Zeit später auch mit Kursen in Wien. Von Beginn an ist die ESWT-Anwendung bei Cellulite dabei integraler Bestandteil der Stoßwellentherapieausbildung (• Abb. 9). 


\section{Fazit für die Praxis}

- Die ESWT nutzt akustische Wellen, um biologische Wirkungen im Zielgewebe zu erzielen.

- Die ESWT löst neben anderen biologischen Effekten eine Stammzellaktivierung aus. Darüber hinaus sind eine Reihe weiterer biologischer Effekte beschrieben, die auch bei der Cellulite eine Rolle spielen können, wie z. B. die Verbesserung der Mikrozirkulation, die Stimulation von LymphVEGF und auch ein antifibrotischer Effekt auf die bei Cellulite fibrosierten Subkutansepten am Tal der Dellen.

- Der Schweregrad einer Cellulite kann sowohl mit der radialen wie auch der fokussierten ESWT positiv verändert werden.

- Es besteht ein enger Zusammenhang zwischen der Anzahl der ESWTBehandlungen und der klinischen Effektivität.

- Auch die Stimulation von autologem Fettgewebe mit „adipose-derived stem cells (ASCs)“ durch die ESWT insbesondere vor Liposuktion ist mit einigen experimentellen Studien belegt und vielversprechend, um die Qualität und die Vitalität von ASCs zu verbessern.

- In der Hand eines sachkundigen Anwenders ist die extrakorporale Stosswellentherapie (ESWT) eine nebenwirkungsfreie Therapieform, die auch in der Kombination mit anderen Therapieverfahren sinnvoll ist.

\section{Korrespondenzadresse}

\section{Prof. Dr. Karsten Knobloch, FACS}

SportPraxis Prof. Knobloch

Heiligerstr. 3, 30159 Hannover, Deutschland professor.knobloch@sportpraxis-knobloch.de

\section{Einhaltung ethischer Richtlinien}

Interessenkonflikt. K. Knobloch ist amtierender Präsident der deutschsprachigen internationalen Gesellschaft für extrakorporale Stoßwellentherapie (DIGEST e. V.) im Ehrenamt und u. a. Vorstandsmitglied der internationalen Stoßwellenfachgesellschaft ISMST (International Society for Medical Shockwave Treatment). Des Weiteren erhielt der Autor als Referent Honorare von Storz Medical für Vorträge. Als
Buchautor erhielt der Autor Honorare von den Verlagen Level10, Unimed Verlag, Springer Verlag, Thieme Verlag, Spitta-Verlag.

Dieser Beitrag beinhaltet keine vom Autor durchgeführten Studien an Menschen oder Tieren. Alle Patienten, die über Bildmaterial oder anderweitige Angaben innerhalb des Manuskripts zu identifizieren sind, haben hierzu ihre schriftliche Einwilligung gegeben. Im Falle von nicht mündigen Patienten liegt die Einwilligung eines Erziehungsberechtigten oder des gesetzlich bestellten Betreuers vor.

Open Access Dieser Artikel wird unter der Creative Commons Namensnennung 4.0 International Lizenz (http://creativecommons.org/licenses/by/4.0/deed. de) veröffentlicht, welche die Nutzung, Vervielfältigung, Bearbeitung, Verbreitung und Wiedergabe in jeglichem Medium und Format erlaubt, sofern Sie den/die ursprünglichen Autor(en) und die Quelle ordnungsgemäßnennen, einen Link zur Creative Commons Lizenz beifügen und angeben, ob Änderungen vorgenommen wurden.

\section{Literatur}

1. Holfeld J, Tepköylü C, Reissig C, Lobenwein D, Scheller B, Kirchmair E, Kozaryn R, AlbrechtSchgoer K, Krapf C, Zins K, Urbschat A, Zacharowski K, Grimm M, Kirchmair R, Paulus P (2016) Tolllike receptor 3 signalling mediates angiogenic response upon shock wave treatment of ischaemic muscle. Cardiovasc Res 109(2):331-343

2. Zhang X, Yan X, Wang C, Tang T, Chai Y (2014) The dose-effect relationship in extracorporeal shock wave therapy: the optimal parameter for extracorporeal shock wave therapy. J Surg Res 186(1):484-492

3. Mazzola RF, Mazzola IC (2015) History of fat grafting. From ram fat to stem cells. Clin Plast Surg 42(2):147-153

4. Coleman SR, Mazzola RF (2009) Fat injection. From filling to regeneration. Quality Medical Publishing St. Louis

5. Rennekampff HO, Reimers K, Gabka CJ, Germann G, Guinta RE, Knobloch K, Machens HG, Pallua $N$, Ueberreiter K, von Heimburg D, Vogt PM (2010) Möglichkeiten und Grenzen der autologen Fetttransplantation - "Consensus Meeting" der DGPRÄC in Hannover, September 2009. Handchir Mikrochir Plast Chir 42(2):137-142

6. Rezek D (2015) Stellenwert der autologen Fetttransplantation in der Brustheilkunde. J Ästhet Chir 8:50-56

7. Cehn YW, Wang JR, Liao X, Li SH, Xiao LL, Cheng B, Xie GH, Song JX, Liu HW (2017) Effect of suction pressures on cell yield and functionality of the adipose-derived stromal vascular fraction. J Plast Reconstr Aesthet Surg 70(2):257-266

8. Raabe O, Shell K, Goessl A, Crispens C, Delhasse Y, Eva A, Scheiner-Bobis G, Wenisch S, Arnhold $S$ (2013) Effect of extracorporeal shock wave on proliferation and differentiation of equine adipose tissue-derived mesenchymal stem cells in vitro. Am J Stem Cells 2(1):62-73

9. Schuh CM, Heher P, Weihs AM, Banerjee A, Fuchs C, Gabriel C, WolbankS, Mittermayr R, Redl H, Rünzle D, Teuschl AH (2014) In vitro extracorporeal shock wave treatment enhancs stemness and preserves multipotency of rat and human adipose-derived stem cells. Cytotherapy 16(12):1666-1678
10. Priglinger $E$, Schuh CMAP, Steffenhagen $C$, Wurzer C, Maier J, Nuernberger S, Holnthoner W, Fuchs C, Suessner S, Rünzler D, Redl H, Wolbank S (2017) Improvement of adipose tissue-derived cells by low-energy extracorporeal shock wave therapy. Cytotherapy 19(9):1079-1095

11. Priglinger $E$, Sandhofer $M$, Peterbauer $A$, Wurzer C, Steffenhagen C, Maier J, Holnthoner W, Nuernberger S, Redl H, Wolbank S (2018) Extracorporeal shock wave therapy in situ-novel approach to obtain activated fat graft. J Tissue Eng Regen Med 12(2):416-426

12. Prigliner $E$, Wurzer $C$, Steffenhagen $C$, Maier J, Hofer V, Peterbauer A, Nurnberger S, Redl H, Wolbank S, Sandhofer M (2017) The adipose tissue-derived stromal vascular fraction cells from lipedema patients: are they different? Cytotherapy 19(7):849-860

13. Cianfarani F, Toietta G, Di Rocco G, Cesareo E, Zambruno G, Odorisio T (2013) Diabetes impairs adipose tissue-derived stemm cell function and efficiency in promoting wound healing. Wound Repair Regen 21(4):545-553

14. SiemsW, Grune T, Voss P, Brenke R(2005) Anti-fibrosclerotic effects of shock wave therapy in lipedema and cellulite. Biofactors 24(1-4):275-282

15. Knobloch K, Joest B, Krämer R, Vogt PM (2013) Cellulite and focused extracorporeal shock wave therapy for non-invasive body contouring: a randomized trial. Dermatol Ther (Heidelb) 3(2):143-155

16. Knobloch K, Kramer R (2015) Extracorporeal shock wave therapy (ESWT) for the treatment of cellulite-a metaanalysis. Int J Surg 24(Pt B):210-217

17. Hexsel D, Camozzato FO, Silva AF, Siega C (2017) Acoustic wav therapy for cellulite, body shaping and fat reduction. J Cosmet Laser Ther 19(3):165-173 
Hier steht eine Anzeige.

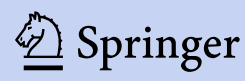

